\title{
Nueva distribución de Paralomis birsteini Macpherson, 1988 en aguas antárticas (Anomura, Lithodidae, Lithodinae)
}

\author{
Patricio M. Arana E. ${ }^{1}$ y Marco Antonio Retamal ${ }^{2}$ \\ ${ }^{1}$ Escuela de Ciencias del Mar, Universidad Católica de Valparaíso \\ Casilla 1020, Valparaíso, Chile \\ E-mail: parana@ucv.cl \\ ${ }^{2}$ Departamento de Oceanografía, Universidad de Concepción \\ Casilla 2407 Apartado 10, Concepción, Chile \\ E-mail: marretam@udec.cl
}

Recibido 25 agosto 1999; versión corregida 2 noviembre 1999; aceptado 8 noviembre 1999.

RESUMEN: Se analizan 88 ejemplares de centolla capturados con trampas en aguas profundas del Océano Austral, próximas al continente Antártico (Subáreas estadísticas 88.3, 48.1 y 48.2). Los ejemplares de Paralomis birsteini Macpherson, 1988, se capturaron en los montes submarinos de Gerlache y en los alrededores de la isla Pedro I, en el mar de Bellingshausen; en el talud continental al oeste de la Península Antártica y en el Mar de Escocia, al norte de las islas Orcadas del Sur, en profundidades entre 621 y 1876 m. Los especímenes presentaron longitudes cefalotorácicas entre 50,8 y $110,2 \mathrm{~mm}$ y peso promedio de $249 \mathrm{~g}$. Los rendimientos, respecto al total de trampas utilizadas en cada subárea, correspondieron a 87,3 g/trampa en la Subárea 88.3; 2,2 g/trampa en la Subárea 48.1; y, 548,8 g/trampa en la Subárea 48.2. Además, se discute la presencia de la familia Lithodidae en el océano Austral.

Palabras claves: Crustacea, Lithodidae, distribución, biología, centolla antártica, Paralomis birsteini, CCRVMA.

\section{New distribution of Paralomis birsteini Macpherson, 1988 in Antarctic waters (Anomura, Lithodidae, Lithodinae)}

\begin{abstract}
Eighty-eight specimens of Paralomis birsteini Macpherson, 1988, were caught with traps over the submarine Gerlache sea mounts and surroundings Pedro I Island, in the Bellingshausen Sea; over the continental slope to the west of the Antarctic Peninsula and in the Scottish Sea (Statistical Subareas 88.3, 48.1 and 48.2), between 621 and $1876 \mathrm{~m}$ depth. Measurements of the cephalothoraxic length of specimens were between 50.8 and $110.2 \mathrm{~mm}$, and 249 -g weight on the average. Yields relative to the total traps used in each subarea, were $87.3 \mathrm{~g} /$ trap in Subarea 88.3; $2.2 \mathrm{~g} /$ trap in Subarea 48.1; and, $548.8 \mathrm{~g} /$ trap in Subarea 48.2. The presence of the family Lithodidae in the Southern Ocean is discussed.
\end{abstract}

Key words: Crustacea, Lithodidae, distribution, biology, Antarctic stone crab, Paralomis birsteini, CCAMLR.

\section{INTRODUCCIÓN}

Las "centollas" constituyen uno de los grupos de mayor relevancia entre los crustáceos decápodos que habitan en aguas australes. Algunas de estas especies presentan interés comercial, siendo explotadas en forma permanente, como es el caso de Lithodes santolla (Molina, 1782) y Paralomis granulosa (Jacquinot, 1847), tanto en aguas jurisdiccionales chilenas como argentinas (Hernández y Díaz, 1986; Lovrich, 1997). Por otra parte, P. spinosissima (Birstein y Vinogradov,
1972) y en menor cantidad P. formosa (Henderson, 1888) han sido capturadas, sólo en algunas temporadas en los alrededores de la isla Georgia del Sur (Otto y MacIntosh, 1996; Watters, 1997).

La familia Lithodidae en aguas australes de los océanos Atlántico y Pacífico fue revisada por Macpherson (1988a, 1988b), quien describió doce especies nuevas para ese sector, entre las cuales se encuentra Paralomis birsteini. Los dos ejemplares 
que permitieron su descripción están depositados en el National Museum of Natural History (Smithsonian Institution, USNM) y del Zoological Museum de la Universidad de Moscú (ZMUM). Esta especie fue dedicada al Dr. Y.A. Birstein por su importante contribución al conocimiento de los Litódidos.

El presente trabajo tiene por objetivo describir la presencia de P. birsteini en aguas profundas del Mar de Bellingshausen, cerca de la península Antártica y en el Mar de Escocia. Además, se incluye algunos aspectos biológico-pesqueros y se actualiza la distribución geográfica y batimétrica de esta especie y de la familia Lithodidae en el océano Austral.

\section{MATERIALES Y MÉTODOS}

El material utilizado en la presente investigación se obtuvo en la prospección de recursos pesqueros realizada a bordo del PAM "Tierra del Fuego" en aguas del océano Austral, entre el 9 de febrero y el 23 de marzo de 1998 (Arana y Vega, 1998). El área de estudio correspondió principalmente al talud continental y únicamente en algunos casos se incluyó la plataforma en torno al continente Antártico e islas adyacentes, en las Subáreas estadísticas 88.3 (Mar de Bellingshausen), 48.1 (Península Antártica) y 48.2 (Mar de Escocia), todas ellas en el ámbito de competencia de la Comisión para la Conservación de los Recursos Vivos Marinos Antárticos (CCRVMA).

En total se calaron 300 trampas (121 en la Subárea 88.3, 138 en la Subárea 48.1 y 41 en la Subárea 48.2) en 52 estaciones de muestreo (Fig. 1), en un rango de profundidad entre 290 y $1920 \mathrm{~m}$. De este total se perdieron únicamente 6 trampas (2\%). Se utilizaron trampas de forma piramidal truncada $(82$ x 55 x $32 \mathrm{~cm})$ y tetragonales $(78 \times 55$ x 40 $\mathrm{cm})$, con bocas o entradas construidas con varillas de fierro de $6 \mathrm{~mm}$ de sección, las rectangulares de $27 \times 10 \mathrm{~cm}$ y las circulares de $30 \mathrm{~cm}$ de diámetro. Como carnada se utilizó aproximadamente $1 \mathrm{~kg}$ de sardina española (Sardinops sagax) enteras y saladas, y calamar entero (Illex argentinus), cada una de estas carnadas en forma separada o mezcladas, envuelta en un trozo de paño de red. El esfuerzo aplicado totalizó 3450 horas de reposo, con un promedio de 11,5 horas por trampa (D.E. = 3,75).

En cada estación se registró la posición, hora de calado y virado del aparejo, profundidad, características del fondo, tipo de carnada y captura obtenida, en unidades y peso. El tiempo medio de reposo de las trampas se determinó como el lapso transcu- rrido desde el momento que se estimaba la llegada de la trampa al fondo hasta el virado de las mismas. La captura por unidad de esfuerzo (CPUE), se determinó como el cociente entre la captura obtenida y el número de trampas caladas, expresada en términos de gramos por trampa (g/trampa).

A los ejemplares capturados se le determinó el sexo y fueron medidos y pesados individualmente. La longitud cefalotorácica (Lc) se midió en línea recta sobre el eje medio dorsal, desde el arco postocular hasta el límite posterior del caparazón utilizando un medidor vernier con precisión $\pm 1 \mathrm{~mm}$. Las distribuciones de frecuencia de tallas se establecieron agrupando los ejemplares en clases de 4 $\mathrm{mm}$ de longitud cefalotorácica. El peso de los individuos se determinó empleando balanzas de $2 \mathrm{~kg}$ $\pm 2 \mathrm{~g}$ de precisión. Además, un cierto número de ejemplares fue congelado para su análisis en tierra.

\section{RESULTADOS}

\section{Descripción de los ejemplares}

Paralomis birsteini Macpherson, 1988 (Fig. 2)

Material examinado:

7 machos, Lc: 50,8-54,0-61,6-75,5-79,0-79,4-81,0 mm

1 hembras, Lc: $61,5 \mathrm{~mm}$

1 hembra ovífera, Lc: 66,9 mm

Diagnosis genérica: El género se caracteriza por presentar un caparazón más o menos pentagonal o piriforme. Las regiones son convexas y están bien definidas. La región gástrica es más prominente que las otras, la región cardíaca tiene forma triangular y está separada de la región gástrica por un profundo surco; el surco cervical está escasamente marcado y es muy poco aparente entre las áreas branquiales y la región cardíaca.

El rostro está formado por una espina basal y al menos un par de espinas dorsales más o menos divergentes dirigidas hacia arriba. El segundo segmento abdominal está formado por una sola placa, desde el tercer al quinto segmento abdominal presentan una clara diferenciación, placas bien calcificadas sin nódulos o áreas membranosas. Sobre cada segmento hay una placa mediana, un par de placas laterales y un par de placas marginales. Las placas marginales y laterales sobre el tercer segmento (y muy raramente sobre el cuarto segmento) algunas veces están fusionadas. La región esternal, entre el primer par de pereiópodos, carece de ranura media longitudinal. 


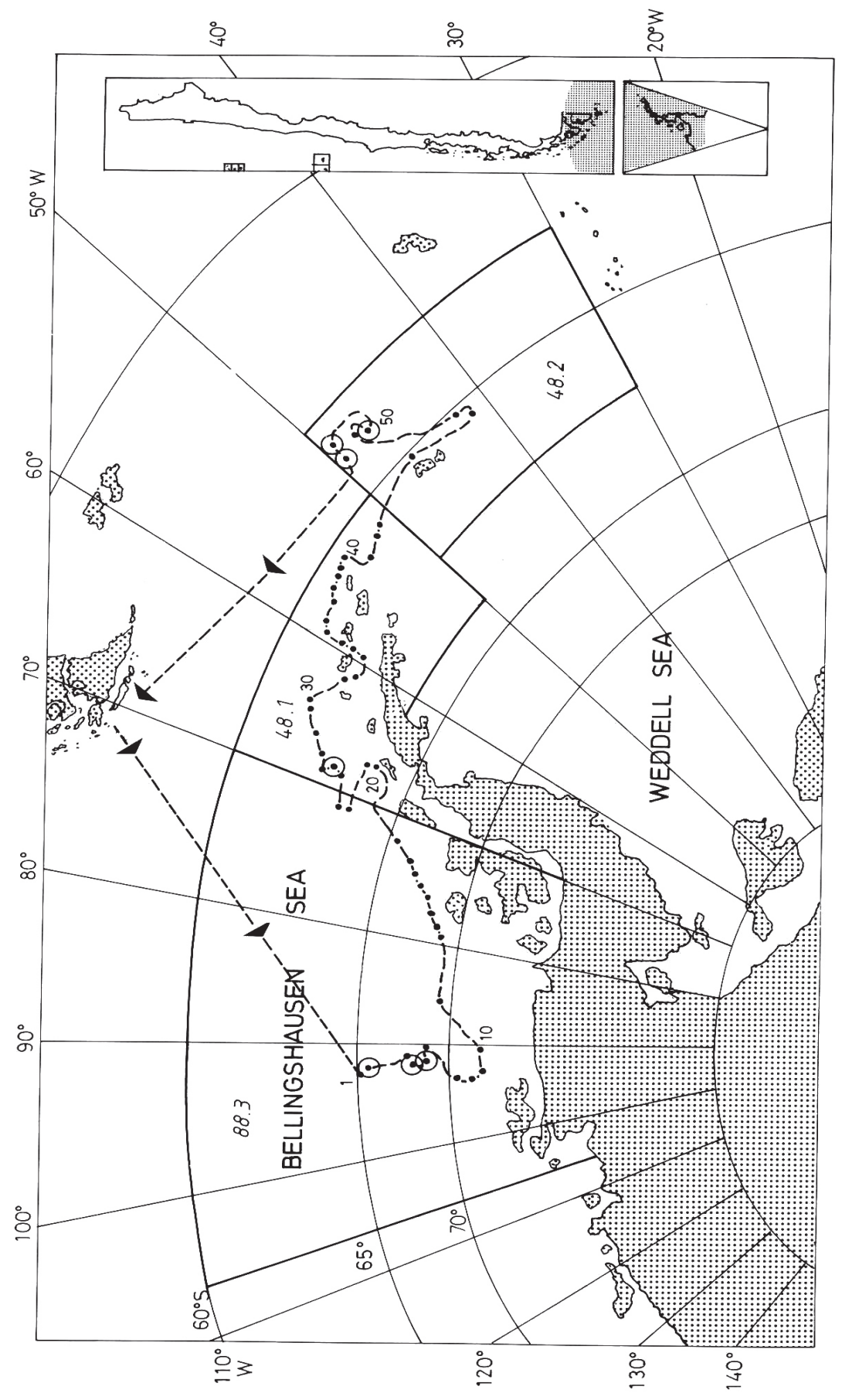

Figura 1. Ruta de navegación (---) y estaciones (•) donde se calaron las trampas (Subáreas 48.1, 48.2 y 88.3). Se indica los lugares donde se capturó Paralomis birsteini $(\odot)$.

Figure 1. Cruise track (---) and stations (•) where pots were set (Subareas 48.1, 48.2 and 88.3). The stations where Paralomis birsteini were caught is indicated $(\odot)$. 
Los ojos presentan córneas pigmentadas. El escafocerito más o menos espinulado está siempre presente.

Los quelípodos son subiguales en longitud, el quelípodo derecho es más robusto, los bordes cortantes sobre el quelípodo derecho lleva dientes redondeados y robustos sobre los dos tercios proximales; el tercio terminal está cubierto por un área córnea. El quelípodo izquierdo lleva dientes pequeños, mientras que redondeados sobre la región proximal y una región córnea, más o menos denticulada, sobre la región terminal. Los dedos están ligeramente curvados hacia el interior, provistos de numerosos manojos de setas. Las patas caminadoras son más o menos alargadas. El segundo pereiópodo es algo más largo que el primero y el tercero.

Diagnosis específica: la superficie dorsal del caparazón lleva gránulos más o menos agudos que no forman verdaderas espinas. El caparazón, quelípodos y pereiópodos están densamente cubiertos con gránulos simples de diferentes tamaños, nunca en manchones. La superficie del caparazón dorsal lleva muchos gránulos de diferentes tamaños pero, nunca aplanados sino redondeados. Los bordes anterior y posterior de las patas caminadoras llevan filas, escasamente definidas, de espinas curvas. Sin crestas sobre los bordes terminales del meropodito y carpopodito del quelípodo. Los bordes laterales del caparazón llevan numerosas espinas o crestas denticuladas. El borde posterior del propodito de las patas caminadoras presentan espinas más o menos robustas. La espina central del escafocerito tiene una o dos pequeñas espinas sobre el margen interno. Las espinas sobre el borde dorsal del carpo del quelípodo no forman una cresta. Existe una concavidad sobre los bordes laterales del caparazón justo bajo el nivel del surco gastrocardíaco.

\section{Aspectos generales}

En la pesca exploratoria se capturaron 88 ejemplares de Paralomis birsteini en 11 de las 104 estaciones realizadas $(10,6 \%)$. La presencia de esta especie se registró en los montes submarinos de Gerlache y en los alrededores de la isla Pedro I, en el mar de Bellingshausen (Subárea 88.3); en el talud continental, en el costado oeste de la Península Antártica (Subárea 48.1) y en el Mar de Escocia, al norte de las islas Orcadas del Sur (Subárea 48.2).

En las trampas donde se extrajo esta centolla, se capturó además de $P$. birsteini, "bacalao antártico" (Dissostichus mawsoni Norman, 1937), "gadimorena" (Muraenolepis microps Lönnberg, 1905 y Muraenolepis sp.), "centolla patache" (Neolithodes diomedeae (Benedict, 1894)) e individuos de Lysianassidae (Amphipoda) (Tabla 1). Ade-

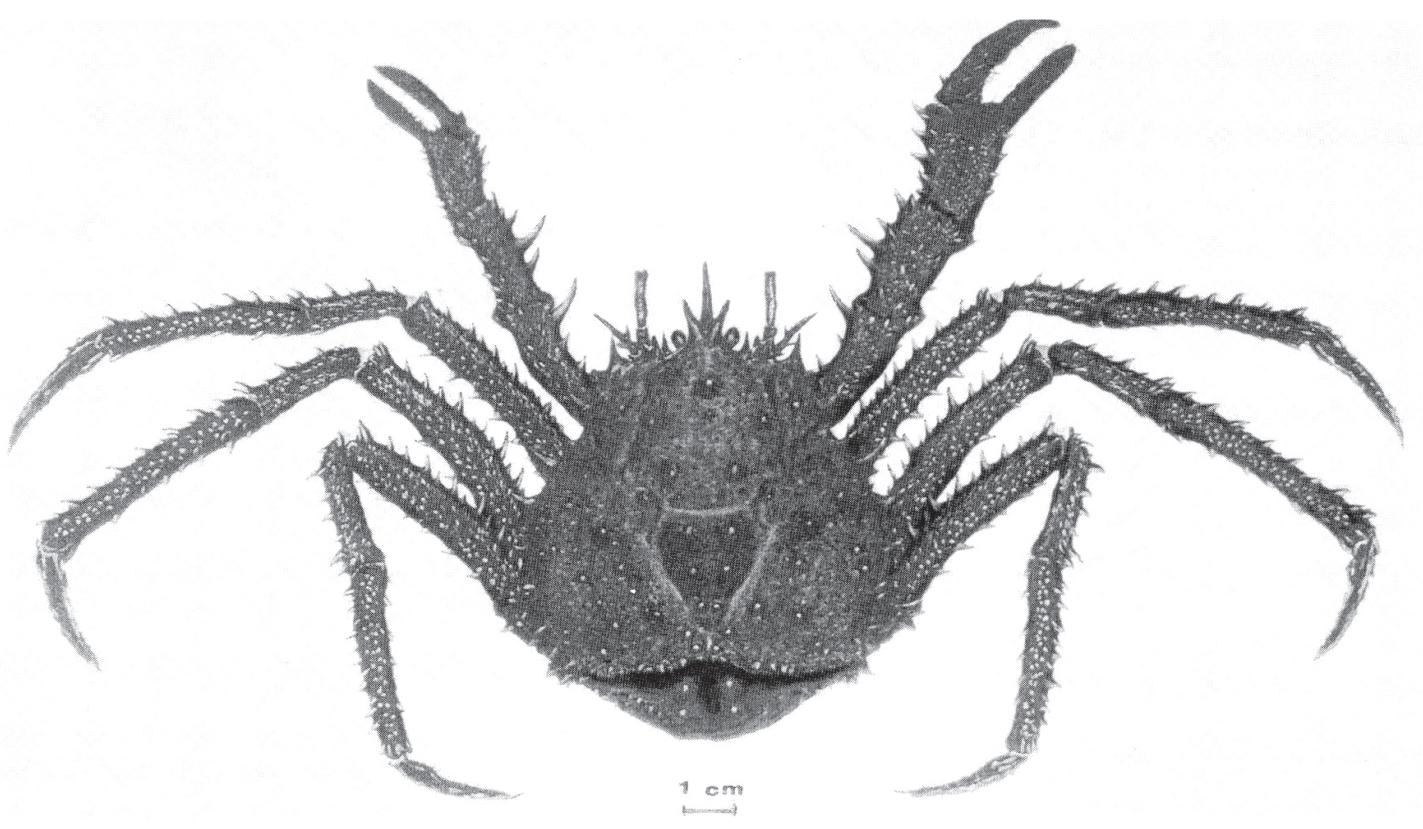

Figura 2. Centolla antártica, Paralomis birsteini Macpherson, 1988.

Figure 2. Antarctic stone crab, Paralomis birsteini Macpherson, 1988. 


\begin{tabular}{|c|c|c|c|}
\hline 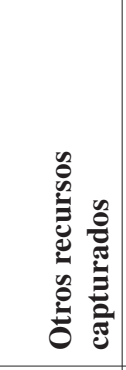 & $\begin{array}{l} \\
\tilde{0}\end{array}$ & 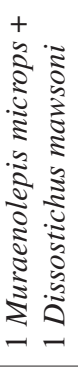 & 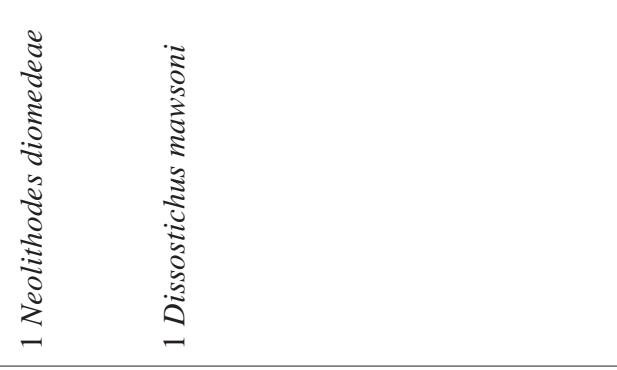 \\
\hline 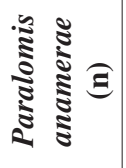 & $n-c$ & N & $n \varrho+-n m$ ก \\
\hline 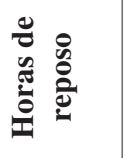 & 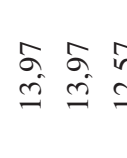 & 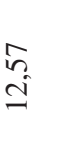 & 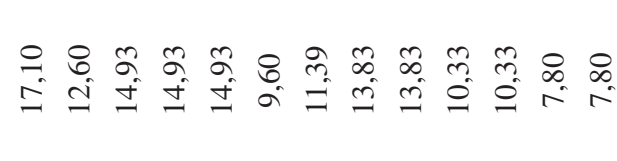 \\
\hline 㺼 & : & ลૂ & 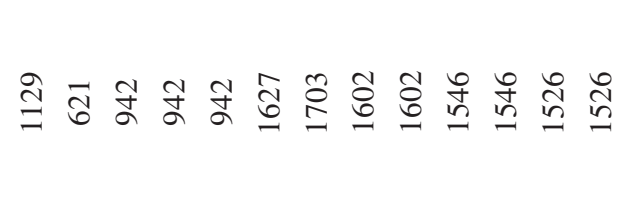 \\
\hline 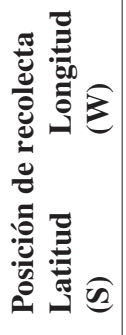 & 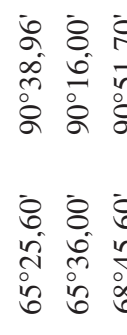 & 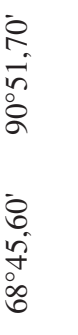 & 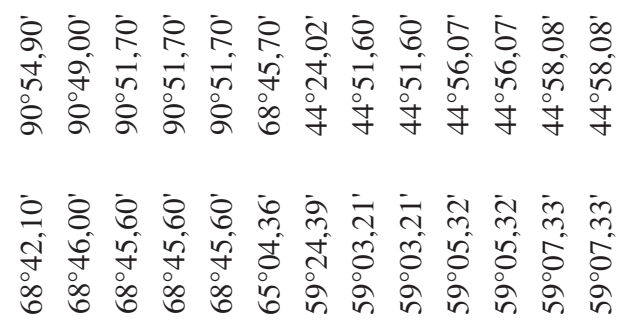 \\
\hline 践 & $a y+$ & $\forall$ & 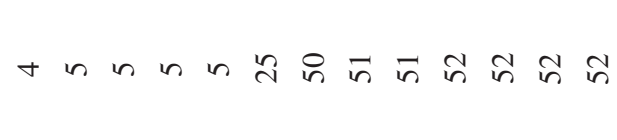 \\
\hline 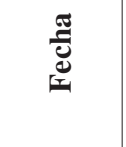 & 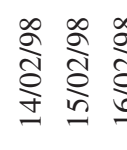 & 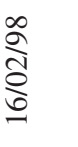 & 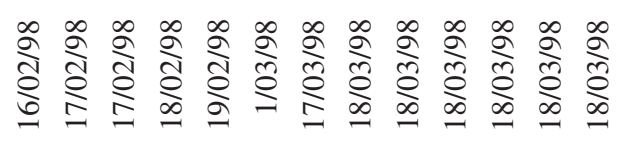 \\
\hline ڤ్̃ & 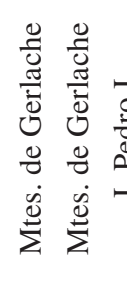 & 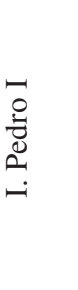 & 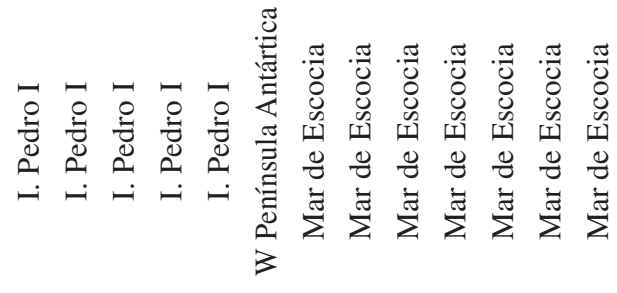 \\
\hline 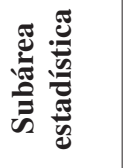 & $\begin{array}{ccc}m & m & 1 \\
\infty & \infty & \alpha \\
\infty & \infty & \alpha\end{array}$ & $\begin{array}{c}m \\
\infty \\
\infty \\
\infty\end{array}$ & 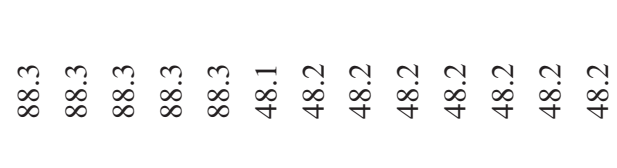 \\
\hline
\end{tabular}


más, en estaciones próximas a las que se capturó $P$. birsteini se encontró "centolla subantártica" (Lithodes turkayi Macpherson, 1988) y el "camarón" Nematocarcinus longirostris (Bate, 1888).

Los ejemplares $P$. birsteini presentaron una captura total de $21,9 \mathrm{~kg}$, con un peso promedio individual de 249 g. La muestra analizada comprendió 77 machos $(87,5 \%)$ y 11 hembras $(12,5 \%)$, de las cuales 10 presentaron huevos en su abdomen $(90,9 \%)$.

La longitud cefalotorácica en los machos estuvo comprendida entre 50,8 y $110,2 \mathrm{~mm}$ con una talla media de $81,1 \mathrm{~mm}($ D.E. $=10,4)$ y en las hembras entre 61,5 y 93,6 $\mathrm{mm}$ y media de $77,0 \mathrm{~mm}$ (D.E. $=10,3$ ). Tanto machos como hembras presentaron modas entre 82 y $90 \mathrm{~mm}$ (Fig. 3).

Los rendimientos de pesca obtenidos en las subárea investigadas, respecto al total de trampas utilizadas en cada una de ellas, correspondieron a 87,3 g/trampa en la Subárea 88.3, 2,2 g/trampa en la Subárea 48.1 y 548,8 g/ trampa en la Subárea 48.2.
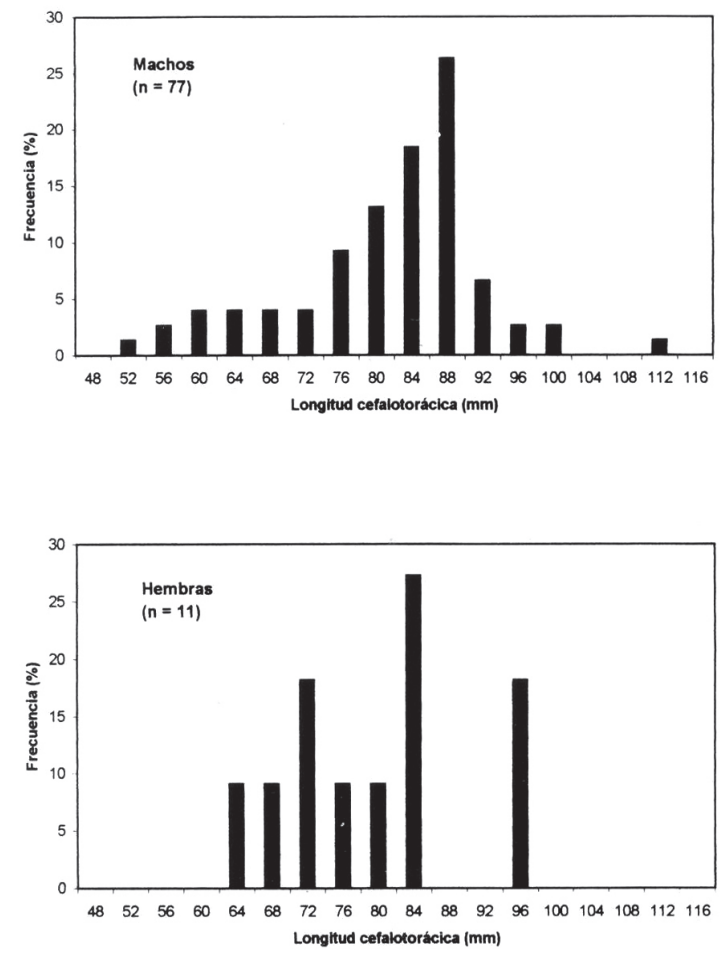

Figura 3. Distribución de frecuencia de tallas de Paralomis birsteini.

Figure 3. Length-frequency distribution of Paralomis birsteini.

\section{DISCUSIÓN}

Entre los organismos capturados con trampas, $P$. birsteini fue la especie con mayor rendimiento individual y con presencia en todas las Subáreas investigadas. La presencia de esta centolla había sido descrita únicamente en el talud en torno a la isla Scott, al norte del Mar de Ross $\left(67^{\circ} \mathrm{S}-180^{\circ} \mathrm{W}\right)$, entre 500 y $1080 \mathrm{~m}$ de profundidad (Macpherson, 1988b). Recientemente ha sido identificado otro ejemplar de esta especie depositado en el Museo de París, el cual fue capturado cerca de la isla Crozet $\left(55^{\circ} \mathrm{S}-4^{\circ} \mathrm{E}\right)$, a $1500 \mathrm{~m}$ (E. Macpherson, com. pers.). De allí que su presencia en los montes submarinos de Gerlache, en los alrededores de la isla Pedro I, en el talud continental al oeste de la península Antártica y en la vecindad de las islas Orcadas del Sur, en el Mar de Escocia, en el rango 621 a $1876 \mathrm{~m}$ de profundidad, amplía considerablemente la distribución geográfica y batimétrica de $P$. birsteini en el océano Austral. Al considerar los lugares y profundidades en que ya se le ha encontrado, es probable que esta especie tenga una distribución circumpolar antártica, entre 500 y posiblemente $2000 \mathrm{~m}$.

Llama igualmente la atención que en estaciones vecinas a donde se capturó $P$. birsteini se encontraron individuos de Lithodes turkayi. Previamente se confundió esta última especie con L. murrayi, cuya distribución finalmente quedó restringida al océano Indico, en las islas Príncipe Eduardo, Crozet (Arnaud, 1971) y Posesión, además del canal de Mozambique, islas Macquarie y al sur de Nueva Zelandia (Yaldwyn y Dawson, 1970), entre 35 y 200 m de profundidad (Macpherson, 1988a). La distribución de L. turkayi ha sido descrita para la zona de las Falkland/Malvinas (Takeda y Hatanaka, 1984; Boschi et al., 1992) y en la costa de Chile (Campodonico y Guzmán, 1972, Revuelta y Andrade, 1978; Retamal, 1981, 1994b), entre 70 a $600 \mathrm{~m}$ de profundidad. Las capturas de $L$. turkayi efectuadas en la presente investigación coinciden con el registro de esta misma especie realizado en la vecindad de la isla Pedro I por Klages et al. (1995), en un rango de profundidad comprendido entre 183 y 257 $\mathrm{m}$. No obstante, en esta oportunidad se encontró esta centolla a mayor profundidad, entre 622 y $678 \mathrm{~m}$ y en otras estaciones hasta los $1696 \mathrm{~m}$. 
Según López-Abellán y Balguerías (1994), tomando en consideración la información contenida en Fischer y Hureau (1985), el límite sur de distribución de la familia Lithodidae estaría alrededor del paralelo $60^{\circ} \mathrm{S}$. No obstante, el haber encontrado a $P$. birsteini y L. turkayi en el Mar de Bellingshausen, se extiende el límite de distribución de esta familia prácticamente hasta los $69^{\circ} \mathrm{S}$ (Tabla 1).

De acuerdo a los resultados globales obtenidos en el crucero, la abundancia de crustáceos fue especialmente baja en la Subárea 48.1 y la más alta en los lances efectuados en la Subárea 48.2. Estos resultados son concordantes con los obtenidos en la campaña ANTARTIDA 8611, en la cual se realizaron 345 lances de arrastres entre 63 y $643 \mathrm{~m}$ sobre las plataformas de las islas y archipiélagos del Mar de Escocia (Subárea 48.2), donde se capturaron sólo 64 ejemplares de centollas, Paralomis spinossisima y P. formosa (Balguerías, 1989; López-Abellán y Balguerías, 1994). Por otro lado, en la pesca exploratoria con trampas realizada por Otto y MacIntosh (1996) en torno a la isla Georgia del Sur (Subárea 48.3), se determinó una gran abundancia de estas especies, con la única excepción de los cañones submarinos cercanos a la isla, donde se encontró escasa abundancia de ambas especies.

En general, en la región del Pacifico y Atlántico analizada, se caracteriza por la presencia de diferentes especies de crustáceos, las que además se distinguen por la abundancia y la profundidad en que se encuentran (Tabla 2). Así, en el extremo austral del continente sudamericano, entre 0 y 300 $\mathrm{m}$ de profundidad, destacan Lithodes santolla y Paralomis granulosa, que constituyen pesquerías tradicionales y de reconocida importancia (Hernández y Díaz, 1986; Lovrich, 1997). Por otra parte, en aguas subantárticas del océano Atlántico Sur, principalmente en los alrededores de la isla Georgia del Sur, se encuentra principalmente $P$. spinossisima y $P$. formosa, con abundancia menor a las especies antes mencionadas y concentradas aproxima-damente entre 200 y $800 \mathrm{~m}$ de profundidad. Finalmente, como se determinó en esta oportunidad, P. birsteini y L. turkayi predominan en aguas profundas (600-1900 m) en la región Pacífico-Atlántico del océano Austral (Subáreas 88.3, 48.1 y 48.2), aunque con densidades bajas. De esta manera, aunque con información aun fragmentaria, se evidencia que la abundancia disminuiría de norte a sur, mientras que la distribución batimétrica aumentaría en igual dirección.
De acuerdo con Bouvier (1896), los Litódidos se habría originado en el Pacífico norte, desde donde se habría irradiado al resto del mundo. Esta idea la sustentó en que la mayor cantidad de especies conocidas se encontró en el océano Pacífico (86\%) y una menor cantidad en el Atlántico (14\%). Esta hipótesis fue reafirmada posteriormente por Makarov (1938), quien postula que desde ese lugar las centollas habrían migrado hacia el oeste del $\mathrm{Pa}$ cífico y hacia el Atlántico a través del estrecho de Bering y océano Artico y por el sur, cruzando por el Estrecho de Magallanes y Cabo de Hornos. Recientemente, Retamal (1994a) sugirió que la teoría de la "sumersión ecuatorial" o "sumersión tropical" explicaría la existencia de diversas especies de la familia Lithodidae en aguas australes del continente sudamericano. Así, en la región ecuatorial habitan a gran profundidad, mientras que hacia la región austral se encuentran en aguas más someras, por cuanto las isotermas en la cual viven están más cerca de la superficie. No obstante lo anterior, llama la atención que al sur del Frente Polar nuevamente la distribución batimétrica de esta familia se profundiza.

De acuerdo a lo señalado, Paralomis birsteini es una de las centollas con distribución más austral hasta ahora conocida, a la vez que es la especie encontrada a mayor profundidad alrededor de la Antártica. Estas características plantean nuevas interrogantes respecto al rol que cumple esta especie en la comunidad de esta región, a la vez que motiva el interés por conocer las adaptaciones fisiológicas de estos animales para vivir en hábitats de condiciones tan extremas.

\section{AGRADECIMIENTOS}

Los autores agradecen a la empresa Pesca Chile S.A. por el apoyo logístico y financiero otorgado para la realización de la presente investigación. Así, también, se reconoce la colaboración brindada por el Capitán, Patrón de Pesca y tripulación del buque espinelero factoría "Tierra del Fuego" durante la realización de la pesca exploratoria. Así, también, los autores desean expresar su más sincero reconocimiento al Dr. Enrique Macpherson M., del Centro de Estudios Avanzados (CSIC), Blanes, Gerona, España, por sus autorizados comentarios y sugerencias. 


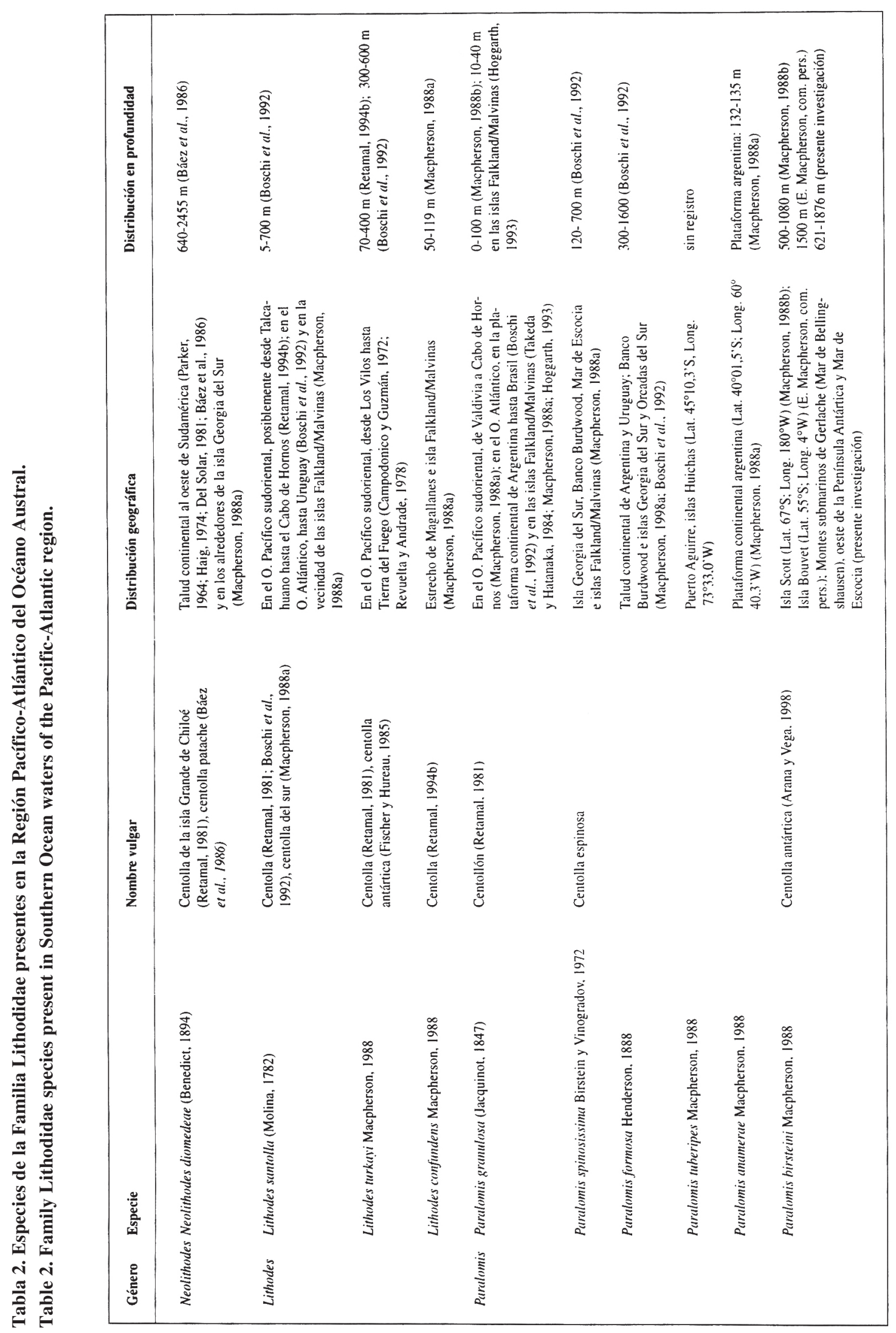




\section{REFERENCIAS}

Arana, P. y R. Vega. 1998. Fishing with pots in the Antarctic region (CCAMLR Statistical Subareas 48.1, 88.2 and 88.3). WG-FSA-98/20: 23 pp.

Arnaud, P. 1971. Lithodes murrayi Henderson, 1988 (Crustacea, Decapoda, Anomura) dans les eaux côtières des îles Crozet (SW de l'Oceéan Indien). Thétys, 3: 167-172.

Báez, P., N. Bahamonde y A. Sanhueza. 1986. Neolithodes diomedeae (Benedict, 1894) (Crustacea, Decapoda, Lithodidae). Invest. Pesq. (Chile), 33: 105-110.

Balguerías, E. 1989. Informe de resultados Antártida 8611. Biología Pesquera. Publ. Inst. Esp. Oceanogr., 2: 267-483.

Balguerías, E. 1990. Las pesquerías antárticas. In: Un viaje a la Antártida, primera expedición científico-pesquera española. Ministerio de Agricultura, Pesca y Alimentación, Instituto Español de Oceanografía, pp. 187-205.

Boschi, E.E., C.E. Fishbach y M.I. Iorio. 1992. Catálogo ilustrado de los crustáceos estomatópodos y decápodos marinos de Argentina. Frente Marítimo, 10: 94 pp.

Bouvier, E.L. 1896. Sur la classification des Lithodinés et sur leur distribution dans les océans. Ann. Scien. Natur., Zoologie, 8: 1.46.

Campodonico, I. y L. Guzmán. 1972. Lithodes murrayi Henderson, 1888, nuevo litódido para la Patagonia austral de Chile (Crustacea, Decapoda, Anomura). Ans. Inst. Pat., Punta Arenas (Chile), 3(1-2): 221-232.

Del Solar, E. 1981. Lithodidae, nueva familia de cangrejos gigantes en el Perú. Bol. Lima, 14: 68-81.

Fischer, W. y J.C. Hureau (eds.). 1985. Fichas FAO de identificación de especies para los fines de la pesca.Océano Austral Areas de la Convención CCAMLR Areas de Pesca 48, 58 y 88. Vol. 1: 232 pp.

Haig, J. 1974. Observations on the Lithodid crabs of Peru, with description of two new species. Bull. Soc. Calif. Acad. Sci., 73: 152-164.
Hernández, M.B. y P. Díaz. 1986. Pesquería de la centolla en la región de Magallanes. In: P. Arana (ed.). La pesca en Chile. Esc. Ciencias del Mar, UCV, Valparaíso, pp. 69-88.

Hoggarth, D.D. 1993. The life history of lithodid crab, Paralomis granulosa, in the Falkland Islands. ICES J. Mar. Sci., 50: 405-424.

Klages, M., J. Gutt, A. Starmans y T. Bruns. 1995. Stone crabs close to the Antarctic continent: Lithodes murrayi Henderson, 1888 (Crustacea, Decapoda; Anomura) off Peter I Island (68 51'S, 9051'W). Polar Biol., 15: 73-75.

López-Abellán, L.J. y E. Balguerías. 1994. On the presence of Paralomis spinosissima and Paralomis formosa in catches taken during the Spanish survey Antártida 8611. CCAMLR Science, 1: 165-173.

Lovrich, G. 1997. La pesquería mixta de las centollas Lithodes santolla y Paralomis granulosa (Anomura: Lithodidae). In: I.S. Wehrtmann y P. Arana (eds.). Biología y pesquería de crustáceos decápodos en aguas templadas de Sudamérica. Invest. Mar., Valparaíso, 25: 41-57.

Macpherson, E. 1988a. Revision of the family Lithodidae Samouelle, 1819 (Crustacea, Decapoda, Anomura) in the Atlantic Ocean. Monogr. Zool. Mar., 2: 9-153.

Macpherson, E. 1988b. Three new species of Paralomis (Crustacea, Decapoda, Anomura, Lithodidae) from the Pacific and Antarctic Oceans. Zool. Scripta, 17: 69-75.

Makarov, V.V. 1938. Fauna of USSR Crustacea. Vol. 9(3): 1-283. (Traducción del ruso, realizada en Jerusalem, Israel).

Otto, R.S. y R.A. MacIntosh. 1996. Observations on the biology of the lithodid crab Paralomis spinosissima from the Southern Ocean near South Georgia. In: High latitude crabs: Biology, management and economics. Alaska Sea Grant College Program Report, 92-02, pp. 627-647.

Parker, R.B. 1964. Zoogeography and ecology of some invertebrates, particularly mollusks, in the Gulf of California and the continental slope off Mexico. Vidensk. Medd. Dansk. Naturh. Foren., 126: 1-178. 
Retamal, M.A. 1981. Catálogo ilustrado de los crustáceos de Chile. Gayana (zool.), 44: 110 pp.

Retamal, M.A. 1994a. Los Lithodidae chilenos. Ans. Inst. Pat. Ser. Cs. Nts., Punta Arenas (Chile), 21: 111-129.

Retamal, M.A. 1994b. Los decápodos de Chile. Universidad de Concepción, Dirección de Docencia, $256 \mathrm{pp}$.

Revuelta, A.G. y H. Andrade. 1978. Nueva localidad para Lithodes murrayi Henderson en el Pacífico Sud Oriental (Crustacea, Decapoda, Anomura, Lithodidae). Not. Mens. Mus. Nac. Hist. Nat. (Chile), 22(261): 3-4.
Takeda, M. y H. Hatanaka. 1984. Records of decapod crustaceans from the Southwestern Atlantic collected by the Japanese Fisheries Research Trawlers. Bull. Nath. Sci. Mus. Tokyo, (A)10: 724.

Watters, G. 1997. Preliminary analyses of data collected during experimental phases of the 1994/ 95 and 1995/96 Antarctic crab fishing seasons. CCAMLR Science, 4: 141-159.

Yaldwyn, J.C. y E.W. Dawson. 1970. The stone crab Lithodes murrayi Henderson: the first New Zealand record. Smithsonian Inst. Press, pp. 550. 